\title{
Energy barriers and mechanisms in solid-solid phase transitions
}

Joost Adam van den Ende, Switzerland, F. Hoffmann-La Roche Ltd

In any manufacturing process control over the properties of the final product is a key factor. In processes where crystalline components are involved, this control necessarily contains producing and maintaining a polymorph with the desired properties, both from an application and a processability perspective. Knowledge about solid-solid polymorphic transitions can be an ingredient in such a strategy.

In the first part of this presentation a combined computational and experimental study of the solidsolid polymorphic phase transitions in DL-norleucine, an amino acid and pharmaceutical model compound, will be discussed. In particular, the energy barriers and mechanisms governing the transition from the low-temperature to the room-temperature polymorphic form will be discussed through the usage of molecular modelling as a computational microscope. The specific modelling techniques used are Nudged Elastic Band (NEB) calculations and steered Molecular Dynamics (MD) simulations. The mechanism of the phase transition is a cooperative movement of hydrogen-bonded bilayers. Probably, local fluctuations in the conformations of the aliphatic chains of the norleucine molecules play a role in transforming the cooperative motion into wave-like behaviour at larger length scales.

The second part of the presentation will contain a general pharmaceutical perspective on the topic of solid-solid phase transitions and their possible influences on the processes involved in drug development and drug production. 